hep-ph 0202108

Kagoshima HE-02-1

June, 2002

\title{
Compressible bag model and the phase structure
}

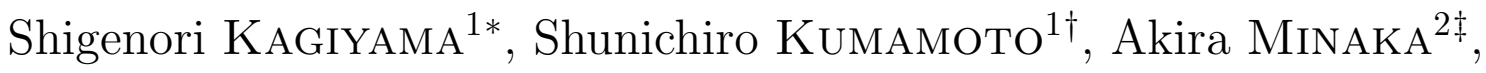

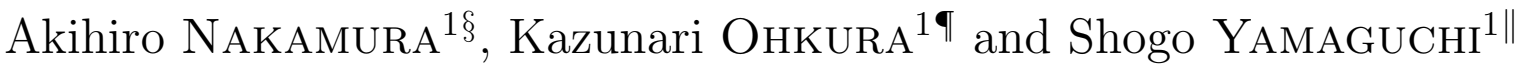 \\ ${ }^{1}$ Department of Physics, Faculty of Science, Kagoshima University, \\ Kagoshima 890-0065, Japan \\ 2 Department of Physics, Faculty of Education, Kagoshima University, \\ Kagoshima 890-0065, Japan
}

\begin{abstract}
The phase structure of hadrons and quark-gluon plasma is investigated by two types of equation of hadron state, namely ideal hadron gas model and the compressible bag model. It is pointed out that, while the ideal gas model produces unrealistic extra hadron phase, the compressible bag model gives an expected and reasonable phase diagram even if rich hadron spectrum is taken into account.
\end{abstract}

\footnotetext{
*e-mail: kagiyama@sci.kagoshima-u.ac.jp

$\dagger$ e-mail: kumamoto@cosmos01.cla.kagoshima-u.ac.jp

$\ddagger$ e-mail: minaka@edu.kagoshima-u.ac.jp

§e-mail: nakamura@sci.kagoshima-u.ac.jp

Ie-mail: ohkura@cosmos01.cla.kagoshima-u.ac.jp

"e-mail: yamaguchi@cosmos01.cla.kagoshima-u.ac.jp
} 


\section{Introduction}

The quark-gluon plasma (QGP) has been expected to appear at high temperature and/or high density, due to asymptotic freedom of QCD. Indeed, not a few of the experimental results upto CERN SPS incident energies suggest the existence of the QGP phase [1], and the recent experiment at BNL RHIC may give us cleaner and richer signals [2].

Theoretically, in the lattice QCD calculations [3, 6, 5, 6, 7], no phase transition is observed for the two light or two light and one medium light flavor cases at zero baryon density. In both cases only a continuous cross over is observed for realistic quark mass values.

On the other hand, in more phenomenological approach, state equations of QGP and hadrons are assumed, and the transition point is determined by Gibbs condition. In this approach, the cross over cannot be reproduced, and that may give rise to a reason to doubt its validity. However, the authors believe that the gross structure of the phase diagram obtained in this approach should still remain valid far away from the cross over region. It should also be noted that at a finite baryon chemical potential, not much is known from lattice QCD because of a well known technical difficulty which makes the Monte Carlo technique inapplicable.

The purpose of the present paper is to investigate what type of the state equation of hadrons is preferable in the phenomenological approach, where the discussion is devoted exclusively to gross phase structure of hadrons and QGP, neglecting the multi-quark states. It means that the detail structure around phase transition or cross over region is neglected.

In the phenomenological approach, there are following problems concerning the gross structure of phase diagrams. When a simple ideal gas of nucleons is used for the equation of hadron state and MIT bag model is used for the equation of QGP state, hadron phase appears at high density and at zero temperature (see, e.g. [\#). This is because

$$
p_{h}^{0} / p_{q}^{0} \rightarrow 27 \quad \text { as } \quad \mu_{B} \rightarrow \infty, \quad(T=0)
$$

where $p_{h}^{0}$ and $p_{q}^{0}$ denote pressures of ideal nucleon phase and (ideal) quark phase, respectively, and $\mu_{B}$ is baryochemical potential. To circumvent this difficulty, volume exclusion effects of hadrons of van der Waals type were taken into account (see, e.g. [8, 9], see also [10]). However, the treatments in [8, 9, 10] are thermodynamically inconsistent [1], for example, $n \neq(\partial p / \partial \mu)_{T}, s \neq(\partial p / \partial T)_{\mu}$. Although the formulation in [1] is thermodynamically consistent, it has still a difficulty that pressure diverges as $n \rightarrow 1 / v_{0}$, where $n$ is number density and $v_{0}$ is fixed hadron volume, and acausality emerges (there is no relativistic rigid body) as the authors themselves pointed out. Thus we were led to 'soft core' model, that is the compressible bag model [12, [13]. 
In addition to the difficulty that hadron phase appears at high density and at zero temperature, an equation of simple ideal gas of hadrons again suffers from the same difficulty at another region, that is, at high temperature and zero baryon number density [14]. The argument in [14] is simple. At $\mu_{B}=0$ and in high temperature region, the pressure $p_{q}$ of QGP phase and the pressure $p_{h}$ of hadron phase are given by

$$
p_{q}=g_{q}\left(1 / 90 \pi^{2}\right) T^{4}-B, \quad p_{h}=g_{h}\left(1 / 90 \pi^{2}\right) T^{4},
$$

where $g_{q}$ and $g_{h}$ are the degeneracy factors in each phase and $B$ is a bag constant. When we consider a mixed gas of lower lying nonstrange hadrons, that is, $\pi, \rho, N$ and $\Delta$, the degeneracy factor $g_{h}$ becomes $3+9+(7 / 4)(4+16)=47$ and it exceeds $g_{q}=2 \cdot 8+(7 / 4) 4 \cdot 3=37$. Thus hadron phase appears again. In the compressible bag model, however, the effective degeneracy factor of hadron phase is greatly reduced so that QGP phase is realized at high temperature even if infinitely many nonstrange mesons are taken into account [15].

The authors of [12, 13, 15] suggested that the compressible bag model gives the phase diagram free from above difficulties, but it has not shown explicitly: In [12] the equations of state only at $T=0$ are used for discussing neutron stars, and in [15] used are those at $\mu_{B}=0$ for multiple production in heavy ion collisions. In [13] the phase diagram with finite $T$ and $\mu_{B}$ is calculated, but rich hadron spectrum is neglected.

The unique purpose of the present paper is, then, to show that the compressible bag model indeed gives the naively expected phase diagram in all regions. As far as the authors know, it is the only model that satisfies the followings:

(a) It is built in thermodynamically consistent formalism.

(b) It is valid in very high temperature regions even if rich hadron spectrum is taken into account.

(c) It is explicitly calculable in whole regions, and it gives well-behaved phase diagrams, i.e. there are no extra hadron phase.

Since the hadron level structure affects the phase diagram, we consider the following two models:

model I: Hadron phase consists of $N \bar{N} \pi$ system while QGP phase consists of nonstrange quarks, their anti-quarks and gluons.

model II: Hadron phase consists of a system of 102 species of hadrons of which masses are less than $10.6 \mathrm{GeV}$, except for hadrons with top quark [16]. QGP phase consists of u-, d-, s-, c-, b-quarks and their anti-quarks and gluons. 
Model I is a model with a few hadrons, while model II is an example which includes many hadrons.

While in [15] the continuous level-density function of [17 is used, in this paper the phase diagrams are calculated with real spectrum of hadrons in order to avoid the model dependence. The effect of the cutoff in the hadron spectrum in model II is discussed in the final section.

In section 2, for comparison the phase structure in free point-like model is shown with the above two types of models, where one can explicitly see the difficulties explained in this section. In section 3, the equation of state in the compressible bag model [13, 15] is briefly reviewed, and with the resulting phase diagram it is shown that the difficulty is indeed removed. Final section is devoted to concluding remarks.

\section{A difficulty in the phase structure for models of free point-like hadrons}

In this section, we assume that all hadrons, quarks, and gluons are free point-like objects and discuss their phase structure. For this purpose, we present expressions of pressures of free point-like particles in order to establish notations in the first subsection. On the basis of these expressions, the phase diagram is discussed and a difficulty is pointed out in the second subsection.

\subsection{Pressures for hadron gas and QGP}

Total pressure $p$ of a mixed gas of $N$-species of free point-like particles is given by

$$
p\left(T, \mu_{B}\right)=\sum_{n=1}^{N} p_{n}\left(T, \mu_{n}\right),
$$

where $T\left(\mu_{B}\right)$ is the temperature (baryochemical potential) of the gas and $p_{n}\left(\mu_{n}\right)$ is the pressure (baryochemical potential) of the $n$-th species of particles. The pressure $p_{n}$ is given by

$$
\begin{aligned}
p_{n} & =\eta_{n} g_{n} T \int \frac{d^{3} \boldsymbol{k}}{(2 \pi)^{3}} \log \left[1+\eta_{n} \exp \left(-\frac{E_{n}-\mu_{n}}{T}\right)\right], \\
E_{n} & =\sqrt{\boldsymbol{k}^{2}+m_{n}^{2}} \\
\mu_{n} & =a_{n} \mu_{B},
\end{aligned}
$$


where $\eta_{n}$ is a statistical factor that takes the value -1 for bosons and +1 for fermions, respectively. The quantities $g_{n}, a_{n}$, and $m_{n}$ denote the degeneracy factor, the baryon number, and the mass of the $n$-th species of particles, respectively.

In low temperature region, $p_{n}$ is expanded as follows;

$$
\begin{aligned}
p_{n}= & \frac{g_{n}}{24 \pi^{2}}\left[\mu_{n} k_{n f}^{3}-\frac{3}{2} m_{n}^{2} \mu_{n} k_{n f}+\frac{3}{2} m_{n}^{4} \log \left(\frac{\mu_{n}+k_{n f}}{m_{n}}\right)\right] \\
& +\frac{g_{n}}{12} \mu_{n} k_{n f} T^{2}+\frac{7 \pi^{2} g_{n}}{720}\left[\frac{3 \mu_{n}}{k_{n f}}-\left(\frac{\mu_{n}}{k_{n f}}\right)^{3}\right] T^{4}, \\
k_{n f}= & \sqrt{\mu_{n}^{2}-m_{n}^{2}},
\end{aligned}
$$

when the $n$-th species of particles are fermions. In the above $k_{n f}$ is a fermi momentum. Eq. (2.5) is applicable for $\mu_{n}>m_{n}$. Otherwise the pressure $p_{n}$ vanishes identically.

Now let us present expressions for pressures of hadrons and QGP. As for total pressure $p_{h}$ of a mixed gas of $N$-species of free point-like hadrons, it is given by

$$
p_{h}\left(T, \mu_{B}\right)=\sum_{i=1}^{N} p_{h_{i}}\left(T, \mu_{i}\right),
$$

where $p_{h_{i}}$ follows $\left.(2.2) \sim 2.6\right)$. In the followings we consider two cases for hadronic systems; that is, the model I and the model II which are defined in the introduction.

On the other hand, total pressure of QGP $p_{q}$ is given by

$$
p_{q}\left(T, \mu_{B}\right)=\sum_{j=1}^{N^{\prime}} p_{q_{j}}\left(T, \mu_{j}\right)-B,
$$

in the bag model. Here $p_{q_{j}}$ follows (2.2) (2.6) and $B$ is a bag constant. The index $j$ denotes the species of particles, and we consider two cases, the model I and the model II.

\subsection{Phase structure}

Given the expressions of pressures of hadron phase and QGP phase, we can consider their phase structure. Critical points are determined by the following Gibbs condition;

$$
p_{h}\left(T, \mu_{B}\right)=p_{q}\left(T, \mu_{B}\right)
$$

In Fig. 1, $p_{h}$ and $p_{q}$ are plotted as functions of $\mu_{B}^{4}$, where $T$ is fixed to zero. In Fig. 2, they are plotted as functions of $T^{4}$, where $\mu_{B}$ is fixed to zero. In both figures, calculations are performed for the model I. As shown in Fig. 1, there is no critical point for $B^{1 / 4}>0.1536 \mathrm{GeV}$. For $B^{1 / 4}=0.1536 \mathrm{GeV}$, there is one critical point. For $0.1534 \mathrm{GeV} \leq B^{1 / 4}<0.1536 \mathrm{GeV}$, 


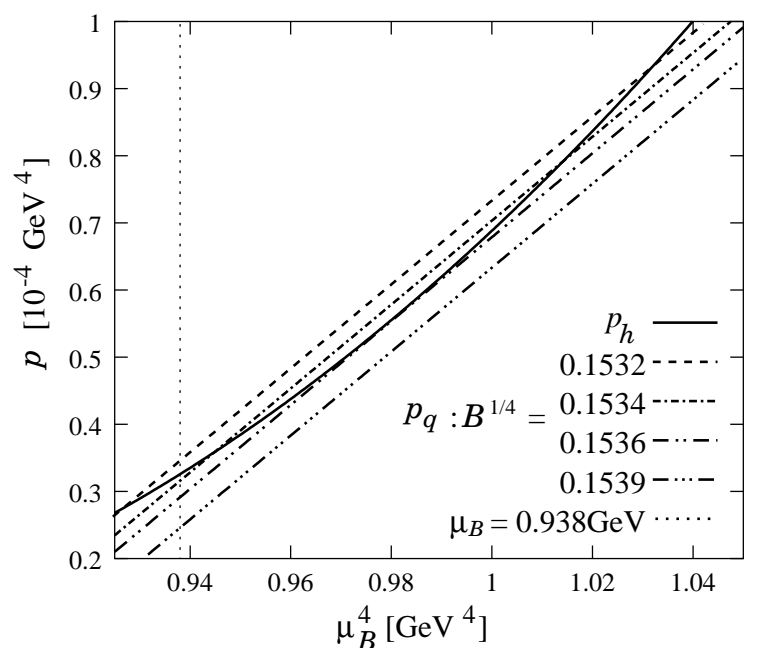

Fig. 1: $p_{h}$ and $p_{q}$ as functions of $\mu_{B}^{4}$ for the model I $(T=0)$. The unit of $B^{1 / 4}$ is $\mathrm{GeV}$.

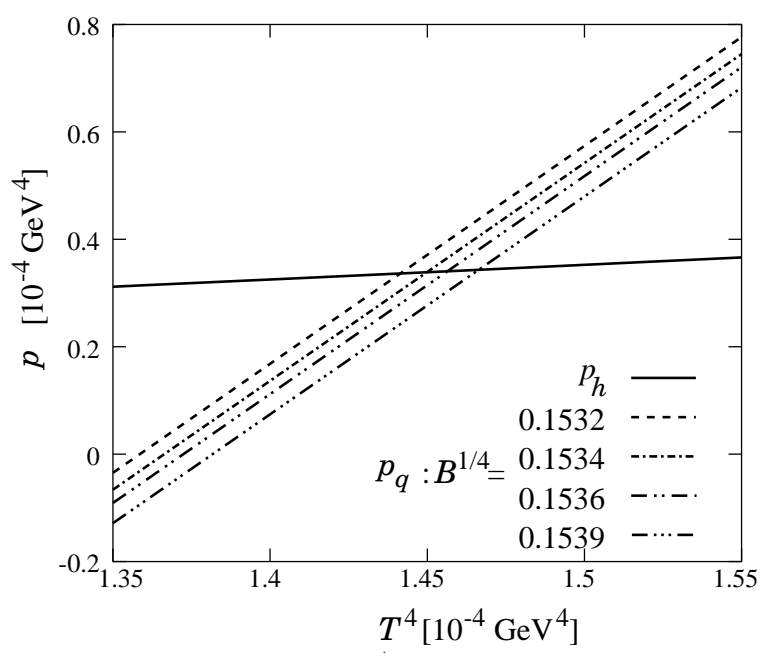

Fig. 2: $p_{h}$ and $p_{q}$ as functions of $T^{4}$ for the model I $\left(\mu_{B}=0\right)$. The unit of $B^{1 / 4}$ is $\mathrm{GeV}$.

there are two critical points. For $B^{1 / 4}<0.1534 \mathrm{GeV}$, there is one critical point since $p_{h}$ vanishes for $\mu_{B}<m_{N}$ and unphysical is an apparent critical point in the region $\mu_{B}<m_{N}$, where $m_{N}$ is nucleon mass. Therefore, at $T=0$, abnormal hadron phase always appears at high densities.

The reason for this difficulty is as follows. In higher density region of $\mu_{B} \gg m_{N}$, masses are negligible so that pressures $p_{h}$ and $p_{q}$ are approximated as

$$
\begin{aligned}
& p_{h} \approx g_{h} \mu_{B}^{4}, \\
& p_{q} \approx g_{q} \mu_{q}^{4},
\end{aligned}
$$

where $g_{h}\left(g_{q}\right)$ is a statistical degree of freedom of hadrons (QGP). Since $\mu_{q}=(1 / 3) \mu_{B},(2.11)$ reads

$$
p_{q} \approx \frac{g_{q}}{81} \mu_{B}^{4}
$$

Then $p_{h}$ becomes large faster than $p_{q}$ as $\mu_{B}$ increases, since an effective statistical degree of freedom of QGP is greatly reduced. In other words, hadrons get a share of energy three times larger than QGP, when $\mu_{B}$ increases, so that $p_{h}$ becomes larger than $p_{q}$. As a consequence hadron phase appears in high density region.

Since the difficulty mentioned above stems from the qualitative nature of the model of hadrons that their statistical degree of freedom is too large, we should consider to modify the model of hadrons. So far we consider the model I at present, the situation will become worse in the model II, since the statistical degree of freedom becomes further large.

In Fig. 3, $p_{h}$ 's for the model I and the model II are plotted as functions of $\mu_{B}$, where $T$ is fixed to zero and in Fig. $4, p_{h}$ 's for those are plotted as functions of $T$, where $\mu_{B}$ is fixed 


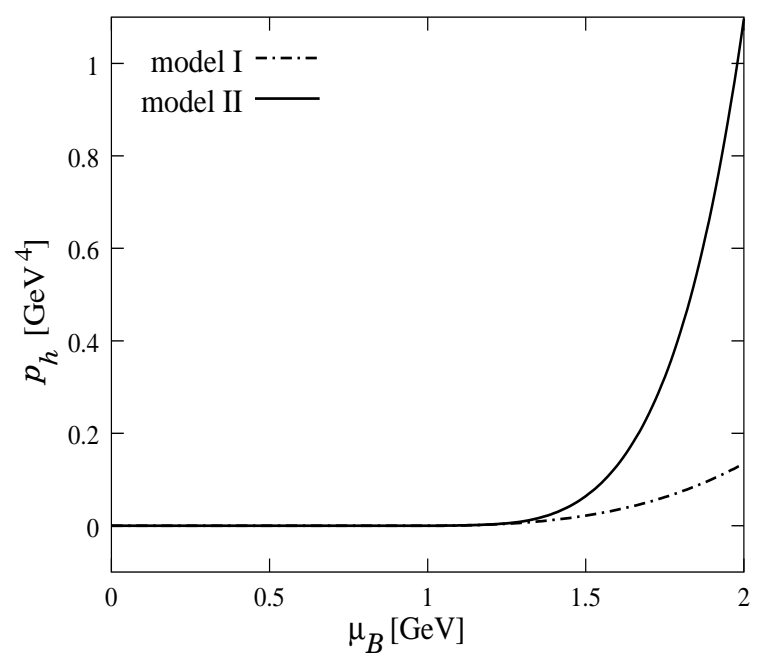

Fig. 3: $p_{h}$ as functions of $\mu$ for the model I and the model II $(T=0)$

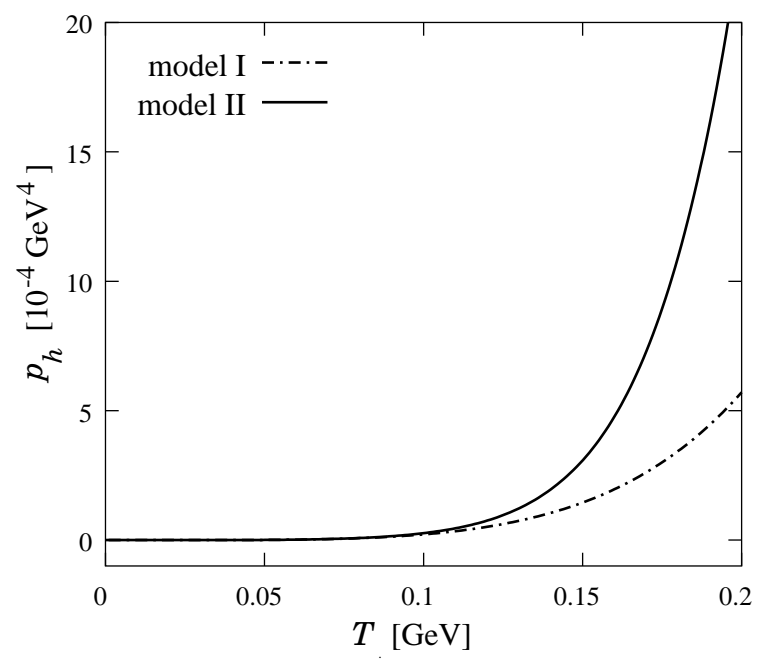

Fig. 4: $p_{h}$ as functions of $T$ for the model I and the model II $\left(\mu_{B}=0\right)$

to zero. As seen in Fig. 3 (Fig. 4), $p_{h}$ for the model II becomes considerably larger than $p_{h}$ for the model I in high density (temperature) region, as expected.

In Fig. $5, p_{h}$ and $p_{q}$ are plotted as functions of $\mu_{B}^{4}$ with $T=0$. In Fig. 6, they are plotted as functions of $T^{4}$ with $\mu_{B}=0$. In both figures, calculations are performed for the model II. Qualitative features in Fig. 1 and Fig. 5 are the same. However, qualitative features in Fig. 2 and Fig. 6 are different. As shown in Fig. 6, hadron phase appears in high temperature region. The resulting phase diagram for the model I [9] is shown in Fig. 7 and that for the model II is shown in Fig. 8. The situation for the model II has become worse than for the model I. In order to modify this situation, we should replace the model of hadrons with alternative ones. As one of them, we formulate the compressible bag model in the next section.

\section{The compressible bag model}

First, we briefly summarize the results of [13, 15]. Let us suppose a mixed gas of $n$-species of hadrons enclosed in volume $V$ at finite temperature $T$. In the compressible bag model, the free energy function $\hat{F}$ of the gas is given by

$$
\begin{aligned}
\hat{F} & =\sum_{i=1}^{n} F_{f}\left(N_{i}, V^{\prime}, T, M_{i}\left(v_{i}\right)\right), \\
V^{\prime} & =V-b \sum_{j=1}^{n} N_{j} v_{j}
\end{aligned}
$$




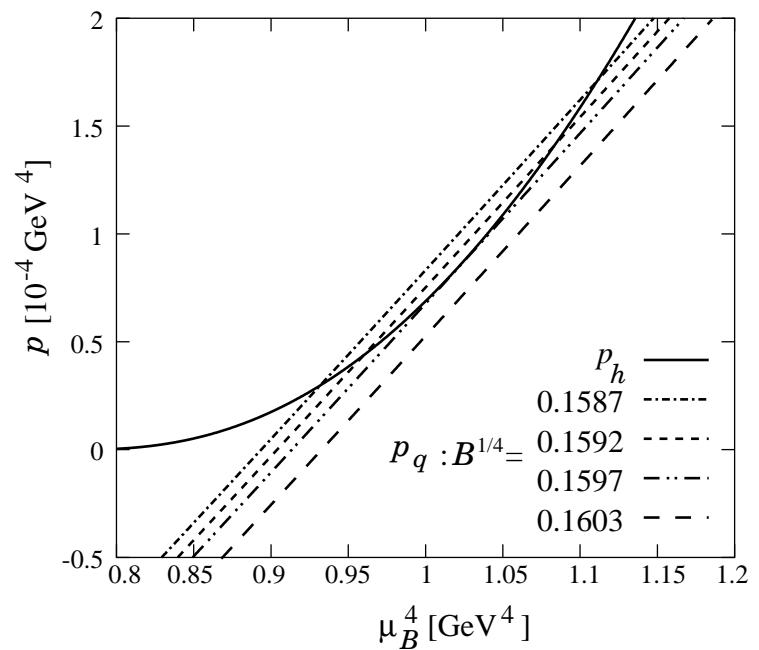

Fig. 5: $p_{h}$ and $p_{q}$ as functions of $\mu_{B}^{4}$ for the model II $(T=0)$. The unit of $B^{1 / 4}$ is $\mathrm{GeV}$.

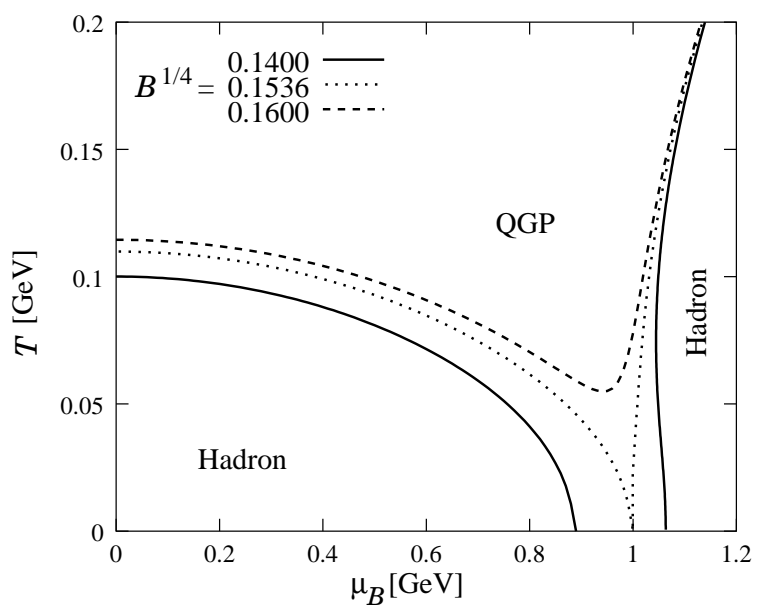

Fig. 7: Phase diagram for the model I. Besides low density and low temperature region, hadron phase appears in high density and low temperature region. The unit of $B^{1 / 4}$ is $\mathrm{GeV}$.

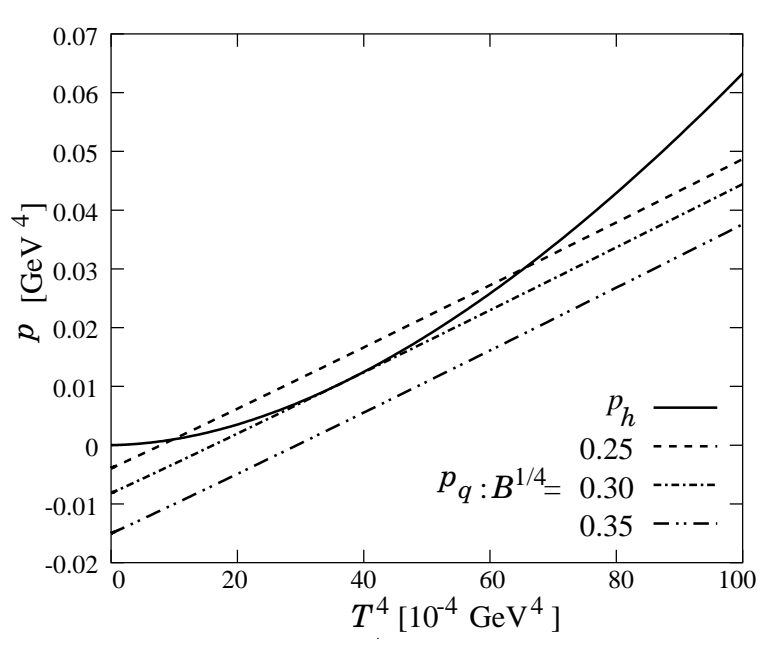

Fig. 6: $p_{h}$ and $p_{q}$ as functions of $T^{4}$ for the model II $\left(\mu_{B}=0\right)$. The unit of $B^{1 / 4}$ is $\mathrm{GeV}$.

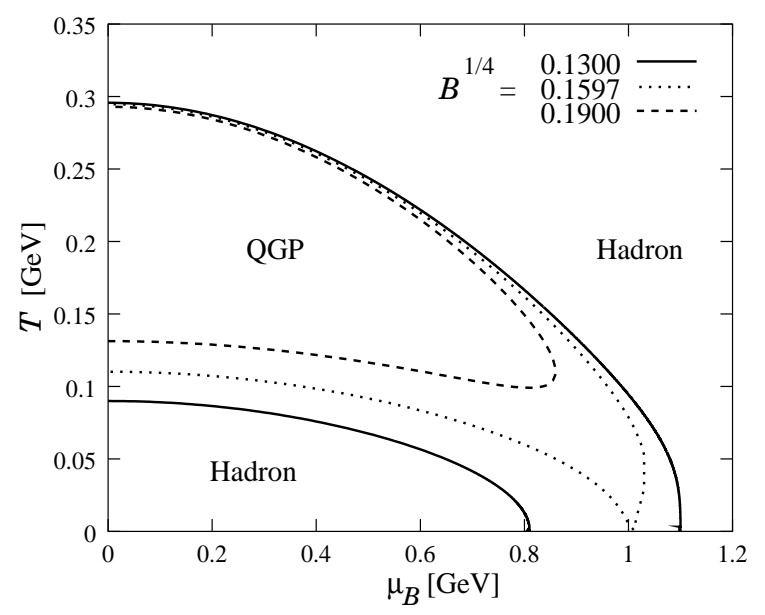

Fig. 8: Phase diagram for the model II. Besides low density and low temperature region, hadron phase appears in high density and high temperature region. QGP phase is realized only in mid density and mid temperature region. The unit of $B^{1 / 4}$ is $\mathrm{GeV}$. 
where $N_{i}$ is a number of $i$-th species of hadrons and $v_{i}$ is its volume and $M_{i}$ its mass. The constant $b$ is a volume exclusion efficiency parameter. The function $F_{f}$ is a free energy function of free point-like hadron gas. As for mass function $M_{i}\left(v_{i}\right)$, we assume that of MIT bag model:

$$
M_{i}\left(v_{i}\right)=A_{i} v_{i}^{-1 / 3}+B v_{i}
$$

where $B$ is bag constant.

Under the approximation that the average of inverse Lorentz factor of $i$-th species of hadrons equals to unity $\left(\left\langle\gamma_{i}^{-1}\right\rangle \approx 1\right)$ in the rest frame of the system, basic requirement of the compressible bag model that $\partial \hat{F} / \partial v_{i}=0$ and the requirement that the chemical potential of hadron should be, $\mu_{i}=\partial \hat{F} / \partial N_{i}=a_{i} \mu_{B}$, where $a_{i}$ is the baryon number of the $i$-th species of hadrons, determine the pressure $p$ of the system as a function of $T$ and $\mu_{B}$ by the following equation:

$$
\begin{aligned}
p= & \sum_{i=1}^{n} \eta_{i} g_{i} T \int \frac{d^{3} \boldsymbol{k}}{(2 \pi)^{3}} \log \left\{1+\eta_{i} \exp \left[-\left(E_{i}-\mu_{i}^{\prime}\left(p, m_{i}\right)\right) / T\right]\right\}, \\
& E_{i}=\sqrt{\boldsymbol{k}^{2}+M_{i}\left(p, m_{i}\right)^{2}} \\
M_{i}\left(p, m_{i}\right)= & m_{i}\left(1+\frac{3 b p}{4 B}\right)\left(1+\frac{b p}{B}\right)^{-3 / 4}, \\
\mu_{i}^{\prime}\left(p, m_{i}\right)= & \mu_{i}-b v_{i} p=a_{i} \mu_{B}-\frac{b m_{i} p}{4 B}\left(1+\frac{b p}{B}\right)^{-3 / 4},
\end{aligned}
$$

where $g_{i}$ is a degeneracy factor of the $i$-th species of hadrons and $m_{i}=4\left(A_{i} / 3\right)^{3 / 4} B^{1 / 4}$ is its mass in the vacuum.

Here two comments are in order. First $p$ determined as above depends on $b$ and $B$ only in the combination of $b / B$ since $M_{i}$ and $\mu_{i}^{\prime}$ depend on $b$ and $B$ in that combination as seen in (3.5) and (3.6). Second the pressure in the compressible bag model does not become so large in high density and/or high temperature region in contrast to the one in the model of free point-like hadrons. This is because masses of hadrons become large in high density and/or high temperature region in the compressible bag model so that kinetic energies of hadrons increase rather slowly and the pressure of hadrons do not become so large. Thus the compressible bag model have a chance to evade the difficulty discussed in the previous section. In the following, we assure that the difficulty is indeed removed, by numerical calculations.

In order to do numerical calculations, we have to fix bag constant $B$ and volume exclusion parameter $b$. The parameter $b$ is determined by the relation

$$
b v_{N}(p=0)=\frac{b m_{N}}{4 B}=\frac{4 \pi}{3}(0.82 \mathrm{fm})^{3}
$$




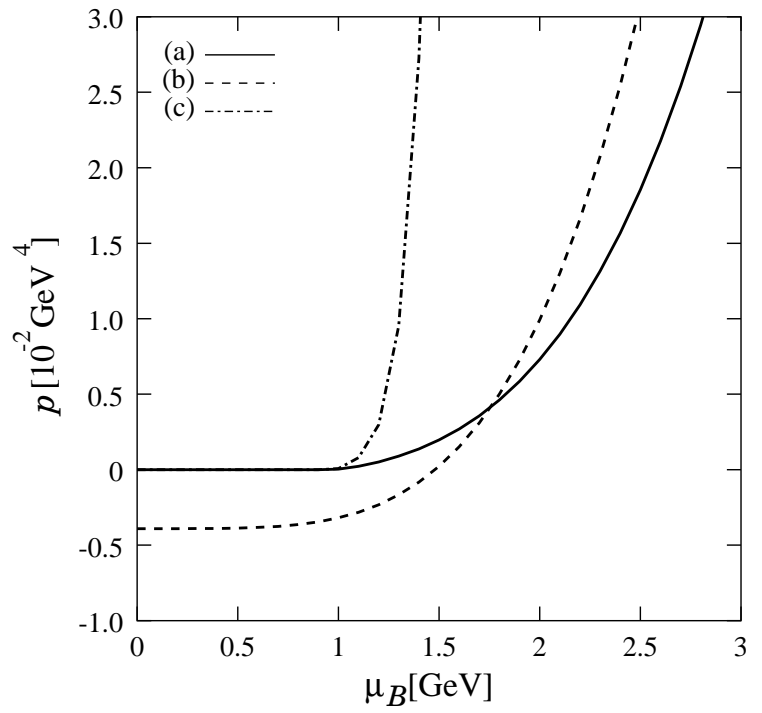

Fig. 9: (a) A solid line shows $p_{h}$ as a function of $\mu_{B}$ for the compressible bag model. (b) A dashed line shows $p_{q}$ for QGP phase. (c) A dash-dotted line shows $p_{h}$ for the free point-like hadron model. All lines are calculated for the model II with $B^{1 / 4}=0.25 \mathrm{GeV}$ and $T=0$.

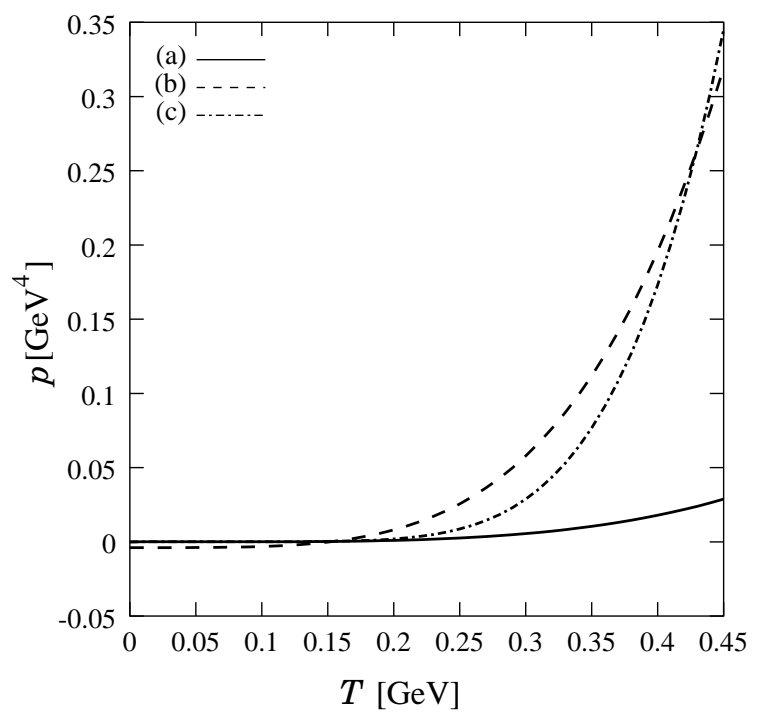

Fig. 10: (a) A solid line shows $p_{h}$ as a function of $T$ for the compressible bag model. (b) A dashed line shows $p_{q}$ for QGP phase. (c) A dash-dotted line shows $p_{h}$ for the free point-like hadron model. All lines are calculated for the model II with $B^{1 / 4}=0.25 \mathrm{GeV}$ and $\mu_{B}=0$.

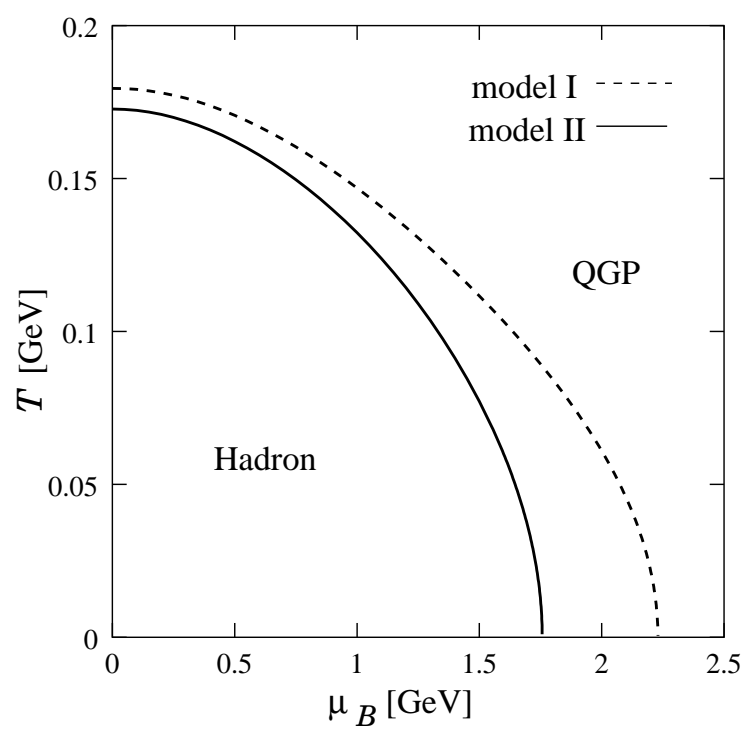

Fig. 11: Phase diagram for the model II (solid line). For comparison, phase diagram is shown for the model I (dashed line). Calculations are done with $B^{1 / 4}=0.25 \mathrm{GeV}$. 
when the bag constant is given. The value $0.82 \mathrm{fm}$ is the proton charge radius. It should be noted that equations of state for hadron phase are determined uniquely at this stage since the equation of states for hadron phase only depend on $b / B$. We tentatively put

$$
B^{1 / 4}=0.25 \mathrm{GeV}
$$

and then get $b=5.0$. The resulting $p_{h}$ and $p_{q}$ are shown as functions of $\mu_{B}(T)$ in Fig. 9 (Fig. 10). We can recognize that the compressible bag model does not suffer from the difficulty that exist for free point-like hadrons. This point is more clearly seen by the phase diagrams shown in Fig. 11 with $B^{1 / 4}=0.25 \mathrm{GeV}$. Critical temperature $T_{c}$ and critical baryochemical potential $\mu_{c}$ are given by

$$
\begin{array}{lll}
T_{c}=0.18 \mathrm{GeV}, & \mu_{c}=2.2 \mathrm{GeV} & \text { in model I, } \\
T_{c}=0.17 \mathrm{GeV}, & \mu_{c}=1.8 \mathrm{GeV} & \text { in model II. }
\end{array}
$$

It is noted that the state equation of hadrons are determined by the ratio $b / B$ and that of QGP by $B$, the critical values, then, depends on $B$ and $b$. If the constraint (3.7) is taken, the values depend only on $B$. As a reference, some cases are shown below;

$$
\begin{array}{lll}
T_{c}=0.14 \mathrm{GeV}, & \mu_{c}=1.7 \mathrm{GeV} & \text { in model I, } \\
T_{c}=0.14 \mathrm{GeV}, & \mu_{c}=1.4 \mathrm{GeV} & \text { in model II, }
\end{array}
$$

for $B^{1 / 4}=0.20 \mathrm{GeV}$ and

$$
\begin{array}{lll}
T_{c}=0.22 \mathrm{GeV}, & \mu_{c}=2.7 \mathrm{GeV} & \text { in model I, } \\
T_{c}=0.21 \mathrm{GeV}, & \mu_{c}=2.1 \mathrm{GeV} & \text { in model II, }
\end{array}
$$

for $B^{1 / 4}=0.30 \mathrm{GeV}$.

From Figs. 9 11 one can see that the compressible bag model gives an expected and reasonable phase diagram in whole regions. This result is stable in the sense that one can choose the bag constant from rather wide range.

\section{Concluding remarks}

In this paper, it has been shown by explicit calculations that the compressible bag model gives well-behaved phase diagram in whole regions, even if many hadron states are taken into account. In the present calculation it is true that, even in the model II, the infinite series of hadrons are cut off at finite mass, but the compressible bag model may give an expected 
phase diagram if infinite series of hadrons are included. This is because, in the model, the masses of hadrons increase and the effect of higher mass state is much suppressed in hightemperature or high-density regions. Indeed in [15] a continuous level-density function are used for hadrons and it is shown that the abnormal hadron phase does not appear in the compressible bag model, although limited at $\mu_{B}=0$.

Free point-like models produce unnatural hadron state in high-temperature or highdensity regions as shown in Sections 1 and 2. A way to avoid this difficulty is the compressible bag model, and it is worth examining in various phenomenological analyses.

\section{References}

[1] Proceedings of the International Conference on Ultra-Relativistic Nucleus-Nucleus Collisions (Quark Matter), Nucl. Phys. A 661 (1999); 638 (1998); 610 (1997).

[2] Proceedings of the 15th International Conference on Ultra-Relativistic Nucleus-Nucleus Collisions (Quark Matter 2001), Long Island, New York, 2001, edited by T.J. Hallman, D.E. Kharzeev, J.T. Mittcell and T. Ullrich (New York, 2002, Elsevier).

[3] Lattice 2000. 18th International Symposium on Lattice Field Theory, Nucl. Phys. B, (Proc. Suppl.) 94, (2001).

[4] Proceedings of the International Workshop Non-Purturbative Methods and Lattice QCD, World Scientific, Singapore (2001).

[5] O. Philipsen, in Lattice 2000, Nucl. Phys. Proc. Suppl. 94, (2001), 49; hep-lat/0011019.

[6] F. Karsh, "Lattice QCD at High Temperature and Density", hep-lat/0106019.

[7] Z. Foder and S.D. Katz, in Lattice 2001, Nucl, Phys. Suppl. 106, (2002), 441; heplat/0110102.

[8] J. Cleymans, K. Redlich, H. Satz, and E. Suhonen, Z. Phys. C 33, 151 (1986).

[9] H. Kouno and F. Takagi, Z. Phys. C 42, 209 (1989).

[10] R. Hagedorn and J. Rafelski, Phys. Lett. 97B, 136 (1980).

[11] D.H. Rischke, M.I. Gorenstein, H. Stöcker, W. Greiner, Z. Phys. C 51, 485 (1991).

[12] S. Kagiyama, A. Nakamura and T. Omodaka, Z. Phys. C 53, 163 (1992); ibid. 56, 557 (1992). 
[13] S. Kagiyama, A. Minaka and A. Nakamura, Prog. Theor. Phys. 95, 793 (1996).

[14] S. Kagiyama and A. Nakamura, XI International Conference on Particle and Nuclei (PANIC '87), Abstract Book II, 456.

[15] S. Kagiyama, A. Minaka and A. Nakamura, Prog. Theor. Phys. 89, 1227 (1993).

[16] Particle Data Group, Eur. Phys. J. C 19, 1 (2000).

[17] J. I. Kapusta, Phys. Rev. D 23, 2444 (1981). . 